\section{Ärztliche Aufklärung in der Radiologie: Fingerspitzengefühl ist gefragt}

Prof. Dr. Peter Wigge, Rechtsanwalt und Fachanwalt für Medizinrecht und Prof. Dr. Dr. Reinhard Loose, Chefarzt am Institut für Radiologie am Klinikum Nürnberg Nord, haben zur ärztlichen Aufklärungspflicht bei diagnostischen Röntgenuntersuchungen aus führliche Informationen zusammengetragen. Warum dieses Thema so wichtig ist und die Radiologie hier eine „Sonderrolle“ einnimmt, erläutern sie im Interview.

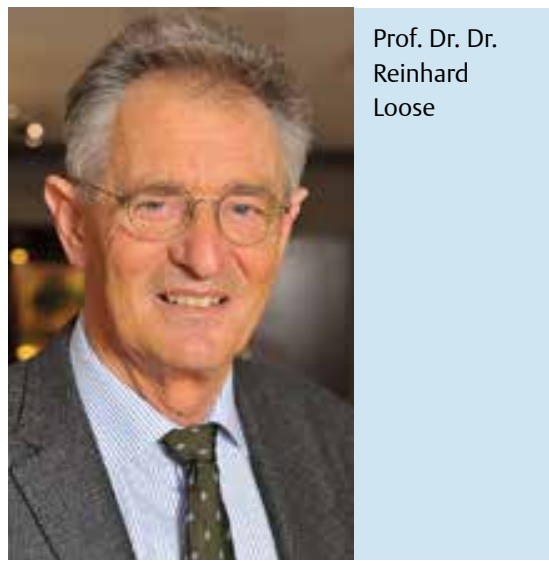

Was hat Sie dazu bewogen, die rechtlichen Aspekte der ärztlichen Aufklärungspflicht in den Blick zu nehmen?

Loose: Die Patientenaufklärung ist in der Radiologie wie in allen anderen Bereichen der Medizin eine wichtige ärztliche Aufgabe. Zeit und Aufwand der Aufklärung müssen aber in einem angemessenen Verhältnis zu den möglichen Risiken einer radiologischen Untersuchung oder eines interventionellen Eingriffs stehen und im täglichen Arbeitsablauf auch zu bewältigen sein. Bis auf wenige Ausnahmen gehört die Diagnostik mit ionisierender Strahlung eher zu den Bereichen des medizinischen Alltags, die mit einem minimalen Risiko behaftet sind.

Wigge: Das Patientenrechtegesetz hat die neuen $\S \S 630 a$ ff. BGB eingeführt, die den Behandlungsvertrag regeln. Durch diese Regelungen wurde jedoch keine Klarheit geschaffen, ob und in welcher Form ein Eingriff durch ionisierende Strahlen vorliegt.

In $\S$ 630e Abs. 1 BGB wird der Arzt verpflichtet, den Patienten über sämtliche für die Einwilligung wesentlichen Umstände aufzuklären. Diese Einwilligung muss gemäß § 630d Abs. 1 S. 1 BGB vor Durchführung einer medizinischen Maßnahme erfolgen. Aus dem Wortlaut dieser Formulierungen könnte sich zunächst eine ufer-

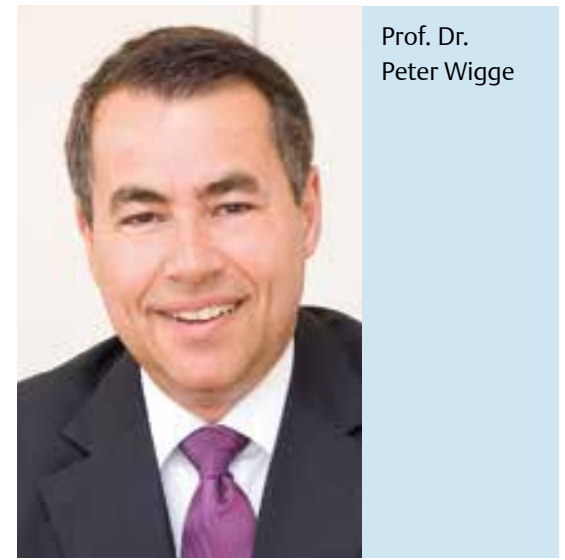

lose Weite aufklärungsbedürftiger Maßnahmen und daraus resultierender Aufklärungspflichten ergeben. Bereits in der Vergangenheit hat die Rechtsprechung hier Grenzen aufgezeigt.

\section{Der Radiologe ist, wie jeder andere} Mediziner auch, verpflichtet, seine Patienten über mögliche Risiken und Folgen der Diagnostik und Therapie aufzuklären. Welche Spezifika zeichnet die Radiologie aus?

Loose: Man muss in der Radiologie $2 \mathrm{Ar}-$ ten von Risiken unterscheiden. Deterministische Risiken beschreiben mögliche Schäden, die in einem Zeitraum von sofort bis zu Wochen oder Monaten nach einer Untersuchung oder einem Eingriff auftreten und damit kausal zuzuordnen sind. Typische Beispiele sind Kontrastmittelzwischenfälle, Blutungen, Infektionen oder bei hohen Eintrittsdosen Strahlenreaktionen der Haut. Über diese Risiken muss zwingend aufgeklärt werden. Stochastische Risiken beziehen sich ausschließlich auf die Anwendung von Röntgenstrahlung im Bereich diagnostischer Dosen und beschreiben die Wahrscheinlichkeit einer Krebsentstehung oder genetischer Schäden. Das Problem ist hierbei 1. die geringe Wahrscheinlichkeit eines Schadens und 2. die Latenz von ca. 15-25 Jahren bis zum Schadenseintritt. Nimmt man bei einer diagnostischen Radiografie eine Dosis von $1 \mathrm{mSv}$ an (typischer Bereich 0,01 bis $1,3 \mathrm{mSv}$ ), so erhöht sich die Wahrscheinlichkeit an Krebs zu erkranken von einer natürlichen Rate von ca. $25 \%$ auf $25,005 \%$. Da die natürliche Krebsrate in Abhängigkeit von Geschlecht, genetischer Disposition, Wohnort und anderen Faktoren eine viel größere Schwankungsbreite zeigt, ist ein kausaler Zusammenhang zwischen diagnostischen Strahlendosen und Krebs nicht herstellbar und eine sinnvolle Aufklärung hierzu kaum möglich.

Wigge: Die Radiologie weist aus juristischer Perspektive verschiedene Spezifika auf. Zunächst ist es ein Fach, welches einerseits eine diagnostische, zum anderen eine interventionelle Komponente beinhaltet. Gerade bei diagnostischen Eingriffen können die Anforderungen an die Aufklärung besonders hoch sein. Zum anderen bleibt, wie oben schon skizziert, die Frage nach der Eingriffsqualität ionisierender Strahlung. Die Sachlage unterscheidet sich hier erheblich von unmittelbaren Schäden wie zum Beispiel in der Chirurgie. Die entsprechenden Primärvorgänge in der Radiologie spielen sich im mikrophysikalischen Bereich ab, mit sehr geringen Schadenswahrscheinlichkeiten nach vielen Jahren. Daher kann eine Kausalität zwischen einer Strahlenexposition und einer manifesten Krebserkrankung nicht hergestellt werden. Die Sterblichkeit durch spontane Krebserkrankungen liegt ca. 500-mal höher als das Risiko eines Karzinoms aufgrund einer CT-Untersuchung mit $10 \mathrm{mSv}$. Die geringfügigen unmittelbaren Strahlenauswirkungen von Röntgenuntersuchungen sind dagegen kaum nachweisbar und innerhalb weniger Tage durch Reparaturvorgänge des Körpers wieder verschwunden. Gerade auch mit Blick auf die natürliche Umgebungsstrahlung kann das Kriterium eines „nicht ganz unerheblichen Gesundheitsschadens“ nicht bejaht werden. Ein solcher ist jedoch nach der Rechtsprechung Voraussetzung einer Haftung.

\section{Wo sehen Sie Schwierigkeiten bei der Aufklärung radiologischer Pati- enten?}

Loose: Zu den Begriffen „Röntgenstrahlung“ und „Krebsrisiko“ haben viele Patienten keine oder nur vage Vorstellungen, während die zuvor genannten deterministischen Risiken besser zu vermitteln 
sind. Es besteht daher das Risiko, dass sinnvolle Untersuchungen und Eingriffe aus unbegründeten Ängsten hinterfragt oder abgelehnt werden. Hier ist das Fingerspitzengefühl des Radiologen gefragt, den Nutzen der Untersuchung zu erklären und zu entscheiden, wann, worüber und wie ausführlich er aufklärt, ohne einen Patienten zu „verschrecken“. Eine Aufklärung sollte das primäre Ziel haben, einen Patienten sinnvoll zu informieren. Nachgeordnet ist der Schutz des behandelnden Arztes vor juristischen Ansprüchen.

Wigge: Die Schwierigkeiten liegen zudem darin, dass die Patienten den Radiologen oftmals nicht als „ihren Arzt“ wahrnehmen und der radiologischen Untersuchung nicht die Bedeutung beimessen wie dem Handeln ihres unmittelbar tätigen Chirurgen oder Internisten. Des Weiteren sind die komplexen Vorgänge in der Radiologie dem Patienten schwieriger zu vermitteln als zum Beispiel Maßnahmen in „schneidenden“ Fächern. Aufgrund der oben geschilderten Vielschichtigkeit der Radiologie ist es für den Radiologen zudem eine besonders schwierige Aufgabe, das rechtlich geforderte „Ob“ und „Wie“ der Aufklärung des Patienten zu bestimmen.

\section{? Wie bewerten Sie die aktuelle} Diskussion über die Delegierbarkeit von Aufklärungspflichten, zum Beispiel auf MTRA?

Wigge: Die MTRA sind sehr qualifiziert für die von ihnen ausgeübten verantwortungsvollen Tätigkeiten. Vom juristischen Standpunkt aus müssen hier aber die gesetzlichen Bestimmungen und die Anforderungen der Rechtsprechung beachtet werden. Die Aufklärung ist Kernbereich ärztlicher Tätigkeit. Aus juristischer Perspektive kann sie somit nicht an MTRA delegiert werden. Grund dafür ist, dass nur der Arzt als derjenige gesehen wird, der auch über mittelbare Folgen und Konsequenzen sowie über Fragen jenseits der konkreten Untersuchung aufklären kann. Dies gilt auch für die Kontrastmittelapplikation, die von MTRA nach der Rechtsprechung allerdings durchgeführt werden darf.

Loose: Genau, die Aufklärung über die genannten deterministischen Risiken ist ärztliche Aufgabe und erfordert die Dokumentation in einem Aufklärungsbogen. Jedoch kann hierbei ein oder eine MTRA bei der Ausfüllung des Bogens und insbesondere der Abfragefelder zu medizinischen Risiken helfen. Auch die Befragung nach einer Schwangerschaft kann an einen oder eine MTRA delegiert werden. Sind alle Risikoabfragen negativ, kann eine Untersuchung ohne weitere Aufklärung durchgeführt werden. Ergeben sich Nachfragen oder Risikokonstellationen, ist es ärztliche Aufgabe, diese mit den Patienten zu besprechen und kann nicht an einen oder eine MTRA delegiert werden.

? In welcher Form ist die Patientenaufklärung in der ärztlichen Ausbildung verankert und wie kann sich der Radiologe up to date halten?

Loose: Im Studium der Medizin ist die ärztliche Aufklärung nach meinem Kenntnisstand nur rudimentär und allgemein verankert. In der Regel findet eine Ausbildung über Wesen und Inhalt der ärztlichen Aufklärung in der Weiterbildung zum Facharzt des jeweiligen Gebiets statt. Für die Radiologie bedeutet dies, dass Ärzte in der Weiterbildung während der Rotation in allen Bereichen der radiologischen Bildgebung und Intervention auch zu den spezifischen Risiken und möglichen Komplikationen zu unterrichten sind.

Wigge: In der RöFo und auf unserer Homepage „Radiologie \& Recht“ (www.radiologie-und-recht.de/) wird immer wieder auf Neuigkeiten aus dem Haftungsrecht, insbesondere auch aus dem Bereich Aufklärung hingewiesen.

\section{? Welchen Nutzen bieten digitale, softwaregestützte Materialien und Formate zur Patientenaufklärung?}

Loose: Sie dürften in Zukunft in weiten Bereichen die papiergebundene Aufklärung ablösen. Der Vorteil der elektronischen Medien besteht darin, dass die bisherigen Aufklärungsbögen sehr einfach in allen benötigten Sprachen angeboten werden können. Weiterhin können zum Untersuchungsablauf Videos mit ergänzenden Erklärungen eingebunden wer- den, die bei Bedarf den Untersuchungsablauf und die notwendige Kooperation des Patienten wesentlich anschaulicher erklären. Auch die elektronische Archivierung und Verfügbarkeit wird durch die elektronischen Medien sicherlich sehr vereinfacht.

Vielen Dank für das Gespräch. 